\title{
Automated reading of a microtitre plate: preliminary evaluation in antimicrobial susceptibility tests and Enterobacteriaceae identification
}

\author{
RJ COURCOL, H DELEERSNYDER, M ROUSSEL-DELVALLEZ, GR MARTIN \\ From the Laboratoire de Bactériologie C, CHR Lille, Hôpital A Calmette, Boulevard du Professeur Leclercq, \\ 59037 Cédex
}

SUMMARY An automated microELISA Reader was evaluated for its ability to read and interpret microtitre plates. A total of 309 microtitre plates were investigated by automated and visual methods. There was disagreement between the methods in one hundred and twelve $(0.6 \%)$ wells. However agreements between the two methods for susceptibility tests and Enterobacteriaceae identification were respectively $98.8 \%$ and $89 \cdot 3 \%$.

Multiwelled plastic trays are used routinely for the determination of minimum inhibitory concentration (MIC) and bacterial identification. The microdilution technique is an accurate and reliable method as compared with a standard reference technique for antimicrobial susceptibility tests 2811121315 and Enterobacteriaceae identification. ${ }^{491017}$ However significant errors can occur in the reading and interpretation of microtitre plates and affect the precision of microdilution tests: ${ }^{2}$ faint haze or small button of growth, identification of the wells with drugs or biochemical characters, transcription errors of laboratory data. An automated system may avoid these difficulties. Recently a manufacturer proposed an automated instrument to read and interpret microtitre plates. ${ }^{6}$ We describe another type of system that allows reading of the microplates and computerisation of the results. This system will be further incorporated in our previously described microcomputer system. ${ }^{5}$

\section{Material and methods}

STRAINS TESTED

Three hundred and nine strains of Enterobacteriaceae were recently clinically isolated and were tested in this study.

MEDIA

Mueller-Hinton broth (Difco Laboratories, Detroit, Mi) was used for antimicrobial susceptibility studies.

Accepted for publication 2 September 1982
Enterobacteriaceae strains were identified with MIC 2000 Enteric Media (Dynatech Laboratories, Alexandria, Va).

ANTIMICROBIAL AGENTS

The different antimicrobial agents were provided by pharmaceutical firms. The antibiotics and the MIC break-point values which are used were

\begin{tabular}{lrl}
\hline Antimicrobial agent & $(\mu g / m l)$ & Abbreviation \\
\hline Ampicillin & $4-16$ & AMP \\
Cephalotin & $8-32$ & CTN \\
Cefamandol & $8-32$ & CFM \\
Cefuroxim & $8-32$ & CXM \\
Moxalactam & $18-24$ & MOX \\
Gentamycin & $4-16$ & GEN \\
Tobramycin & $4-16$ & TOB \\
Netilmicin & $4-16$ & NET \\
Doxycyclin & $4-16$ & DOT \\
Colistin & 2 & COL \\
Ticarcillin & 128 & FIC \\
Cefoxitin & $8-32$ & CXT \\
Cefotaxim & $8-32$ & CTX \\
Mezlocillin & $64-256$ & MEC \\
Kanamycin & $8-32$ & KAN \\
Sisomicin & $4-16$ & SIS \\
Dibekacin & $4-16$ & DKB \\
Amikacin & $8-20$ & AKN \\
Thiamphenicol & $8-32$ & THI \\
Fosfomycin & 32 & FOS \\
Thin & &
\end{tabular}

None of these antimicrobial solutions was sterilised after preparation and there was no bacterial 
contamination. All of the buffers, weighing vials and plastic containers for the solutions were sterile. Antimicrobial susceptibility dilution tests were performed according to the recommendations of the National Committee for Clinical Laboratory Standards. ${ }^{16}$

\section{EQUIPMENT}

The automated system consisted of a microELISA Reader MR-580 (Dynatech Laboratories) connected on-line with an Apple II Plus Microcomputer (Apple Computer Inc, Cupertino, Ca) equipped with RS-232 C computer interface (Leanord, Haubourdin, France). The test wavelength of the reader was equal to $570 \mathrm{~nm}$ and the threshold to $1 \cdot 10$. U-bottom microtitre plates (Dynatech Laboratories) were prepared and inoculated with a MIC 2000 System (Dynatech Laboratories), as described ${ }^{14}$ and used according to the manufacturer's instructions.

\section{PROCEDURES}

The computerised data system was developed for the needs of the laboratory and the application programs were written in BASIC Applesoft by the authors. The wavelength and the interpretation thresholds were? selected after preliminary tests. The 230 most frequent numerical codes of 21 Enterobacteriaceaev species were loaded in the microcomputer memorys eliminating the need for a manual codebook. Othe $\mathbb{D}$ numerical codes could be further added in the program but the small capacity microcomputer core will always limit the quantity of numerical codes.

Microtitre plates were read after 15 to 18 hours of incubation at $37^{\circ} \mathrm{C}$. Readings were taken within 4 . min of the addition of the chemical reagents. The blanking was automatic against sterile distilled watet. contained in the first well. All numerical data were transferred from the reader to the microcompute $\omega$ which separated them into a plus and minus form $₫$ However, results of three biochemical parameters (acetoine production, indole production, $\beta$-galac $=$ tosidase) were typed on the keyboard by the tech $z$ nician because the colours of these reactions did no난 coincide with the test wavelength. The resulf

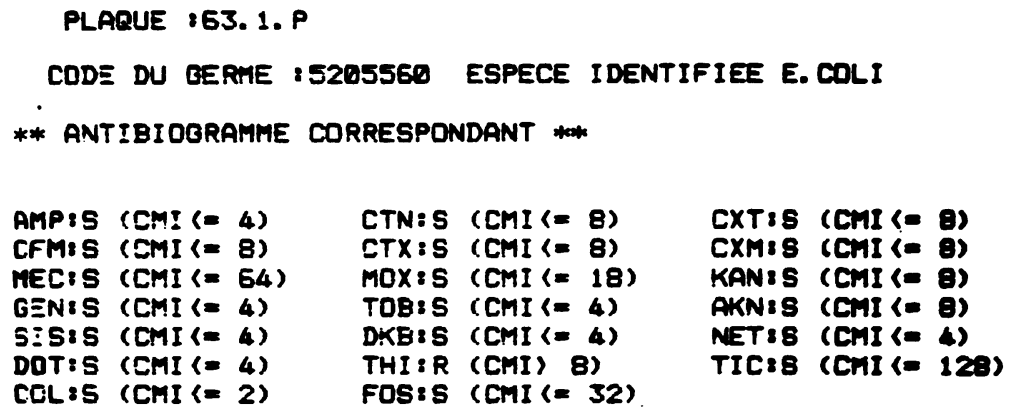

Fig. 1 Example of printout with complete bacterial identification and susceptibility tests.

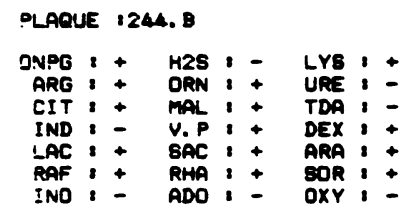

CODE DU BERIE 35336770 ESPECE IDENTIFIEE NON REPERTORIEE WOIR CATAL ** ANTIBIOGRAMIE CORRESPONDANT **

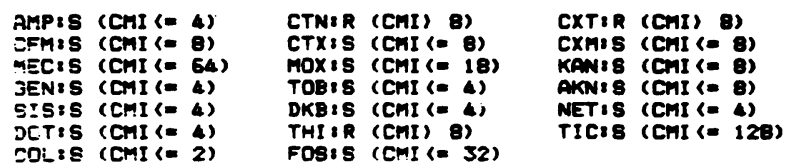

Fig. 2 Example of printout without bacterial identification: biochemical results and numerical code only are printed. 
appeared on the cathode ray tube and was simultaneously printed. Figure 1 represents a printed report which gives the record number of the specimen, the numerical code and the identification of the micro-organism, and the different susceptibilities to the antimicrobial agents. The second printed report ticket (Fig. 2) differs from the first one: the results of 21 biochemical reagents are printed as the numerical code. In this case the identification of the micro-organism does not appear because the numerical code is not programmed and must be compared manually to those reported in the analytical codebook.

The system automatically determined the identification and the susceptibility tests and delivered a printed report in about two minutes. The reading of the microtitre plate and the transmission of the data from the reader to the microcomputer respectively required 1.25 minute and 13 seconds; the results of the three biochemicals were typed in about 10 seconds; the computerisation time ranged from 3 to 4 seconds; the result was printed in 7 seconds.

All microtitre plates were interpreted by a single observer with a standard viewer. The visual reading was considered as the reference method. To exclude bias automatic and visual readings were performed at random.

\section{Results}

A total of 309 microplates were inoculated with 309 Enterobacteriaceae and were independently read with the MicroELISA Reader and a standard viewer. Each microdilution tray contained 20 biochemical reagents and 20 drugs. Consequently, tests with the 309 strains generated 17613 wells with 6180 biochemical characters and 11433 wells representing 6180 different antimicrobial tests.

Of the results obtained with the automated reader, $112(0 \cdot 6 \%)$ wells disagreed with the visual method. With biochemicals reagents discrepancies were observed with $37(0.6 \%)$ wells giving $33(10.7 \%)$ misidentifications.

Seventy-five $(0.6 \%)$ of 11433 susceptibility tests disagreed with the visual reading. Consequently 75 $(1 \cdot 2 \%)$ susceptibility test results differed with the reference reading. The existence of air bubbles or dust on the bottom of the well was respectively observed in 19 cases $(0 \cdot 2 \%)$ and seven cases $(0 \cdot 06 \%)$.

\section{Discussion}

Barry and Braun ${ }^{3}$ reported that the examination of 25022 MICs gave a discrepancy of $5.2 \%$ when measured by two visual readings. This error rate was considered satisfactory by the latter for tests on serial twofold dilutions. Automation and mechanisation of the reading process would help the difficult task of defining certain endpoints. With an automated reader, Ellner and Myers ${ }^{6}$ obtained an agreement equal to $90.0 \%$ between machine and visual bacterial identification.

Our results show that the automated reader and the software developed by the authors are highly reliable when compared to visual reading. Although the accuracy of susceptibility tests is excellent $(98.8 \%)$, the error rate of misidentifications $(10.7 \%)$ demands improvements in the selection of interpretation thresholds. However, this disagreement is close to that found by Ellner and Myers. ${ }^{6}$ The most frequent biochemical reagents with discrepancies were carbohydrates (10 cases) and Moeller's medium (12 cases). These discrepancies may be explained either by a lack of clear cut reaction ${ }^{4}$ or by the reading of the microtitre plate with a single wavelength. The use of the sample filters of the microELISA Reader (five filters) may allow better discrimination of the colour reactions. Thus the typing of three biochemical results on the keyboard should not be necessary because the reactions should be read with the well wavelength. A further difficulty occurred because of air bubbles in the wells during thawing. Formation of these bubbles can be prevented by adding $0.02 \%$ Tween 80 into the sterile distilled water used to prepare the standardised inoculum. ${ }^{6}$

In comparison with a previous study, ${ }^{2}$ the automated reading of susceptibility tests performed with microtitre plates is more accurate than visual reading and may be applicable for routine use. The system avoids transcription errors of laboratory data, improves data management for epidemiological studies, and saves time. The system does not depend on a single commercial supply of test kits allowing its application for testing many antibiological reagents.

We are very grateful to Miss BC Wanat for patient secretarial aid and to $\mathrm{Mr}$ John Hall for improving the English of this manuscript.

\section{References}

' Athar MA, Evans LD, Edney LG. Biochemical identification and microdilution MIC testing of clinically isolated micro-organisms using a semi-automated (Dynatech MIC 2000) System. Can J Med Technol 1978;40:183-6.

${ }^{2}$ Barry AL. Broth dilution techniques. In: The antimicrobic susceptibility test: principles and practices. Philadelphia: Lea \& Febiger, 1976:95-9.

${ }^{3}$ Barry AL, Braun LE. Reader error in determining minimal inhibitory concentrations with microdilution susceptibility test panels. J Clin Microbiol 1981;13:228-30.

${ }^{4}$ Courcol RJ, Husson MO, Izard DE, Martin GR. Comparison of the MIC 2000 Enteric Media with API 20 E and conventional 
methods for identification of Enterobacteriaceae. Zentralbl Bakteriol [Orig A] 1982 (in press).

${ }^{5}$ Courcol RJ, Roussel-Delvallez M, Martin GR. A microcomputer system for clinical bacteriology: experience of 12 months' trial. $J$ Clin Pathol 1982;35:881-7.

- Ellner PD, Myers DA. Preliminary evaluation of the Auto-Scan-3, an instrument for automated reading and interpretation of microdilution trays: identification of aerobic Gram-negative bacilli. J Clin Microbiol 1981;14:326-8.

${ }^{7}$ Fung DYC, Miller RD. Rapid procedure for the detection of acid and gas production by bacterial cultures. Appl Microbiol 1970;20:527-8.

${ }^{8}$ Gavan TL, Town MA. A microdilution method for antibiotic susceptibility testing: an evaluation. Am J Clin Pathol 1980;53:880-5.

${ }^{9}$ Gavini F, Leclerc H. Quality assurance of multiple well system for the mechanical identification of Enterobacteriaceae strains. IASM Dallas, March 1981. Archiv für Lebensmittel Hygiene 1983 (in press).

${ }^{10}$ Huhtanen CN, Naghski J, Dellamonica ES. Microfermentation series for identification of single colonies of Enterobacteriaceae. Appl Microbiol 1972;24:618-27.

" Jones RN, Barry AL, Bigelow J, et al. Evaluation of the MICUR System for quantitative antimicrobial susceptibility testing: a multiphasic comparison with reference methods. J Clin Microbiol 1982;16:153-63.

${ }^{12}$ Mac Lowry JD, Marsh HH. Semi-automatic microtechnique for serial dilution-antibiotic sensitivity testing in the clinicat laboratory. J Lab Clin Med 1968;72:685-7.

${ }^{13}$ Mac Lowry JD, Jaqua MJ, Selepak ST. Detailed methodolog $\vec{F}$ and implementation of a semi-automated serial dilution microtechnique for antimicrobial susceptibility testing. App Microbiol 1970;20:46-53.

${ }^{14}$ Mac Master PR, Robertson CA, Witebsky FG, et al. Evaluation ot a dispensing instrument (Dynatech MIC 2000) for preparin microtitre antibiotic plates and testing their potency during storage. Antimicrob Agents Chemother 1978;13:842-4.

${ }^{15}$ Marymont JH, Wentz RM. Serial dilution antibiotic sensitivity testing with the microtitrator system. Am J Clin Pathab 1966;45:548-51.

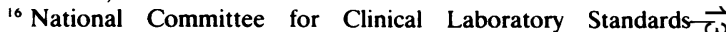
Proposed Standards PSM-7. Standard methods for dilutio antimicrobial susceptibility tests for bacteria which grow aerobically. Villanova, Pa: NCCLS, 1980.

17 Proietti A, Piacentini I. Prove biochimiche di identificazion batterica: presentazione di un metodo miniaturizzato in piastreo microtitre. Quad Sclavo Diagn 1978;14:117-26.

Requests for reprints to: Dr RJ Courcol, Laboratoire de. Bactériologie C, CHR Lille, Hopital A. Calmette Boulevard du Professeur Leclercq, 59037 Lille Cedexi France. 